\title{
Spatial distortions induced by multiple visual landmarks: How local distortions combine to produce complex distortion patterns
}

\author{
THOMAS SCHMIDT \\ University of Göttingen, Göttingen, Germany \\ STEFFEN WERNER \\ University of Idaho, Moscow, Idaho \\ and \\ JÖRN DIEDRICHSEN \\ University of California, Berkeley, California
}

\begin{abstract}
Visual landmarks introduce systematic distortions into spatial short-term memory for single target positions, the exact form of the distortion depending on the spatial layout of the landmarks. In two experiments, we investigated how the combined effect of two landmarks can be predicted from the effects of individual landmarks. Participants used a mouse cursor to reproduce the positions of briefly presented targets in the context of one, the other, or both landmarks. We found that distortions near a landmark are independent of whether another landmark is present, so that remembered space is partitioned into regions dominated by single landmarks. Interestingly, the display midpoint behaves like a "virtual landmark," with its own pattern of distortion. Results are inconsistent with current models of spatial memory distortions but suggest that attentional processes can lead to enhanced fidelity of salient regions in topographical neural networks while also introducing some spatial biases.
\end{abstract}

Many vision scientists agree that "space is special"that the spatial location of an object is not just one feature among others, like its color, form, or orientation. Instead, it is the spatial arrangement of features that defines complex objects or scenes (Palmer, 1999). The brain's major coding strategy for representing space is to use topographical maps where adjacent features in physical space tend to be encoded in adjacent areas of the neuronal representation. Starting from retinotopically organized maps like V1 and the early visual areas, spatial maps further downstream the visual pathways can be organized in terms of different frames of reference-for example, in the visual guidance of pointing and grasping movements (Flanders, Helms Tillery, \& Soechting, 1992). Despite our subjective impression of being highly accurate at perceiving spatial relations within a visual scene, there is abundant psychophysical evidence that some stimuli induce dramatic distortions into the mental representation of distances and angles, as exemplified by the familiar visual illusions encountered in two-dimensional line

This work was supported by a grant from the German Science Foundation (We 1973/3) to S.W. We heartily thank our laboratory teams at the University of Göttingen and the University of Idaho. Thanks also to Julia Trommershäuser, Dirk Vorberg, Jochen Müsseler, and Satoru Suzuki for helpful discussions. Correspondence should be addressed to T. Schmidt, University of Göttingen, Institute of Psychology, Gosslerstr. 14, D-37073 Göttingen, Germany (e-mail: thomas.schmidt@ psych.uni-goettingen.de). drawings. Visual illusions commonly consist of some target element being spatially misperceived in the presence of simple nontarget elements-for example, the flanking arrow tips in the Müller-Lyer illusion or the receding "railroad tracks" in the Ponzo illusion.

Besides purely perceptual distortions, spatial distortions induced by simple nontargets also arise in shortterm memory for simple two-dimensional visual stimuli (Crawford, Huttenlocher, \& Engebretson, 2000; Sheth \& Shimojo, 2001; Werner \& Diedrichsen, 2002). In an early experiment, Nelson and Chaiklin (1980) presented a circle with a target dot located on a visible diameter line. The participants viewed the display for $1 \mathrm{sec}$ and then tried to reproduce the target's exact location on the diameter line from memory. Their responses were systematically distorted away from the center of the circle, with an additional distortion away from the circumference line if viewing time was unrestricted. The distortional pattern was sensitive to changes in the geometry of the stimulus: When the circle was changed into an ellipse, errors were more pronounced along the longer axis of the ellipse. Clearly, the presence of the circumference line induced distortions into the spatial memory representation of the target stimuli.

In a similar experiment, Huttenlocher, Hedges, and Duncan (1991; see also Laeng, Peters, \& McCabe, 1998) also presented a single target dot within a large circle, but in contrast to Nelson and Chaiklin's (1980) method, in this 
procedure participants' reproductions were not restricted to a diameter line but were free to depart from the target in any direction. The clustering of responses suggested that participantsencoded stimulus positions in a representation similar to a polar coordinate system centered on the circle midpoint, with independent biases in the angular and radial coordinates. Additionally, participants seemed to spontaneously segment the circle into quadrants on the basis of the subjective horizontal and vertical meridian lines, since responses were systematically biased away from the boundaries between these quadrants.

In previous experiments in our laboratories, we have used an even simpler configuration of only two landmark dots, one in the left and one in the right half of an otherwise empty display (Werner \& Diedrichsen, 2002; Werner \& Schmidt, 2000). This two-landmark configuration is the simplest one having a defined size and orientation. Participants had to remember the exact location of a briefly presented target dot with respect to the landmarks and then use the mouse cursor to reproduce it after a short retention interval. We found that the landmarks induced a systematic pattern of spatial biases, which we call their distortional field: Targets near the landmarks are reproduced too far away from them, and there is an additional bias away from the midpoint between the landmarks. At the same time, landmarks as well as midpoints are regions of markedly reduced variance in reproductions. Distortional fields are strictly dependent on stimulus geometry, closely following translation, rotation, and expansion of configurations consisting of two or three landmarks (Diedrichsen, 1998; Schmidt, 2002; Werner \& Schmidt, 2000).

Several lines of argument clearly relate these effects to memory rather than perceptual processes. When participants reproduce the target position by quick pointing movements rather than the more time-consuming mouse cursor movements, the amount of distortion strongly increases with retention interval, with only slight distortions when reproduction is performed immediately after target offset (Diedrichsen, Werner, Schmidt, \& Trommershäuser, 2003). In order to study even shorter retention intervals, we developed a new task where the target was first presented along with the landmarks, a short masking interval was then introduced, and the target was presented again, but shifted either in or against the direction of expected distortion (Werner \& Diedrichsen, 2002; Werner \& Schmidt, 2000). Participants' judgments of the direction of target shift were biased in the expected direction, indicating that distortion gradually develops over the course of a few hundred milliseconds. Note that although these data clearly show that distortions develop in spatial memory during the retention interval, purely perceptual distortion would not be able to explain the effect, because any distortion in the perception of target position would also apply to the perception of mouse cursor position. Participants would then adjust the misperceived cursor position to match the misperceived target position, so that no measurable discrepancy would result.

The purpose of this series of experiments was to obtain further insight into the formal properties of distor- tional fields. In particular, we wanted to know whether it is possible to predict the properties of two-landmark distortional fields from the fields of single landmarks. This would be an important first step to finding an algorithm relating the distortional fields of arbitrary multilandmark configurations to their simple elements. In the next section, we will show that existing models are not able to predict the distortional field of two landmarks from singlelandmark fields, although they highlight interesting properties such a predictive algorithm might have.

\section{Models of Spatial Memory Distortions}

The Nelson-Chaiklin model. Nelson and Chaiklin (1980) proposed a weighted distortion theory to describe the data patterns obtained in their experiments. In their view, the remembered target position is distorted with respect to the position of landmark stimuli in the visual field (here, all points constituting the circle must be considered a set of landmarks). Distortion is supposed to be toward the nearest landmark because the distance between landmark and target is underestimated according to a power function, so that distortions become stronger with increasing distance from the landmark. In a multiplelandmark display, each landmark contributes to the overall pattern, with a weight nonlinearly decreasing with distance from the target. Therefore, the distortion and weighting effects have opposite impacts on remembered target position. At so-called equilibrium points (e.g., the center of a circle), no distortion occurs because the weighted sum of all distortions is zero. Importantly, the model does not allow for any kind of interactions between landmarks; their effects just seem to combine independently. However, detailed predictions for novel landmark configurations are very difficult to derive from the model because there is no mathematical formulation. Obviously, the authors were concerned with finding an elegant redescription of their data on the basis of known psychophysical results (e.g., Stevens' power law for remembered distance), rather than trying to explain how spatial distortions arise in the first place.

The Huttenlocher et al. model. Huttenlocher et al. (1991) present a more formal approach to account for spatial distortions of remembered visual stimuli. In their prototype model, they assume that participants' responses are not only influenced by their recollection of the target's location but also by the spatial "category" it is assigned to (in their experiments, the quadrant of the circle in which the target is located). Categories are represented by "prototype" locations in the center of the category. Two processes can lead to spatially distorted memory retrieval. First, the restriction that a target must not be remembered in the wrong spatial category leads to truncation of those parts of the memory distribution that overlap category boundaries, resulting in moderate bias away from such boundaries. More importantly, the prototype location is used to supplement the estimate of uncertainly remembered target positions with a weight proportional to the amount of uncertainty, leading to biases toward the category prototype. The model is stated math- 
ematically and allows for an excellent fit to Huttenlocher et al.'s and others' data, which might not be surprising because of a large number of free parameters.

Importantly, the category boundaries and prototypes are derived post hoc from the data pattern, so that any application of the mathematical details of the model depends on having found the layout of category boundaries by inspection of the data. The model thus lacks predictive power for novel landmark configurations, including the issue of how two single landmarks might combine. Interestingly, however, it allows for qualitative changes to emerge when one is advancing from a one-landmark to a two-landmark configuration because new categories and prototypes might arise. Thus, the model allows for distortional fields of single landmarks to combine nonlinearly, with new Gestalt-like properties emerging from the combined configuration that may even depend on the participants' encoding strategies.

The Suzuki-Cavanagh model. Suzuki and Cavanagh (1997) have suggested a biologically plausible explanation for how spatial distortions might arise from attentional effects in topographical neuronal maps. In their experiments, observers were asked to adjust the relative position of two briefly flashed vertical lines until they were perceived as vertically aligned. Shortly before the presentation of the lines, a distractor circle was presented next to one of the lines at one of several distances. Under these conditions, the line was misperceived away from the distractor so that the two lines appeared misaligned. Importantly, the effect was modulated by visual attention because when distractors were placed on both sides of the lines, distortion was away from the attended but not from the unattended distractor. Suzuki and Cavanagh therefore assumed that the brief presentation of the distractor draws attention to this location, which might lead to changes in the spatial coding properties of cells having receptive fields near the focus of attention (Desimone \& Duncan, 1995). In particular, the authors showed that target locations will be misrepresented away from the focus of attention if receptive fields wander toward the attentional spotlight, form a more fine grained spatial representation there, or selectively inhibit cells outside the spotlight. Their model, unlike Huttenlocher et al.'s (1991), does not presuppose the existence of advanced spatial concepts, such as categories or prototypes, but relies merely on lateral interactions between cortical cells. Although this model is designed to account for a perceptual effect, the basic idea can be employed for explaining spatial memory distortions by assuming that spatial attention is applied directly to topographical memory maps and that landmarks and midpoint receive more attention than other locations (i.e., are attended more intensely or more often). Alternatively, distortional effects might be fed forward from perceptual to memory maps as participants continue to direct overt or covert attention to the landmarks visible during the retention interval.

Distortional effects in Suzuki and Cavanagh's (1997) model are locally restricted to the vicinity of the attentional focus. Therefore, the distortional pattern around a landmark should remain unchanged if a second landmark is presented at sufficient distance, provided that the first landmark still has the focus of attention. In a twolandmark display, the landmark closest to the target would be expected to dominate the size and direction of the distortion. In principle, the model also allows for individual coding strategies mediated by the strategic deployment of attention.

\section{Overview of the Experiments}

In Experiment 1, the participants' task was to reproduce the location of a briefly presented target. In order to measure individual and combined fields, the left, the right, or both landmarks were presented in random order, and a model was developed to predict the two-landmarks field from the single-landmark fields. Experiment 2 replicated these findings with a refined laboratory setup designed to minimize extraneous spatial references. It also extended the results to vertical landmark configurations to check whether distortional fields are invariant regarding the display orientation.

\section{EXPERIMENT 1}

\section{Method}

Participants. Six students (21 to 23 years, all female, all righthanded) from the Institute of Psychology at the University of Göttingen participated for course credits or for a payment of $12 \mathrm{DM}$ per hour. Their vision was normal or corrected to normal.

Apparatus. The experiment was controlled by a PC with an AMD K-2 processor (300 MHz). Stimuli were presented on a 14-in. VGA color monitor $(640 \times 480$ pixels [px] $)$ in synchrony with the monitor retrace rate of $60 \mathrm{~Hz}$. Participants were seated at a distance of approximately $100 \mathrm{~cm}$ from the screen so that $1^{\circ}$ of visual angle equaled approximately 35 pixels. Head position was not fixed.

Stimuli. All stimuli were presented against a black background $\left(0.01 \mathrm{~cd} / \mathrm{m}^{2}\right)$. Landmarks were green unfilled circles $\left(28.7 \mathrm{~cd} / \mathrm{m}^{2}\right)$, $11 \mathrm{px}\left(.31^{\circ}\right)$ in diameter and $80 \mathrm{px}\left(2.27^{\circ}\right)$ to the left or right of the screen center. The target was a small white dot $\left(43.0 \mathrm{~cd} / \mathrm{m}^{2}\right)$ with a diameter of $3 \mathrm{px}$, presented at 1 of the 33 possible locations shown in the upper panel of Figure 1, with the restriction that no target was presented at a position currently occupied by a landmark. The entire grid spanned a visual area of about $6.80^{\circ} \times 1.70^{\circ}$. A dynamical mask similar to static interference on a television screen was used that filled the entire screen. It consisted of black and white elements $(2 \times 2 \mathrm{px})$, with one quarter of the elements randomly set to white at any given time. There were four such random patterns in a repetitive sequence such that each array was presented for $33 \mathrm{msec}$ and the entire sequence had a frequency of $7.5 \mathrm{~Hz}$.

Procedure. A trial began with the appearance of the target together with the left, right, or both landmarks (Figure 1, lower panel). Participants were allowed to inspect this stimulus for $1,500 \mathrm{msec}$ under freeviewing conditions, without any restriction of eye movements. The target was then replaced by the dynamic mask for $500 \mathrm{msec}$ while the landmarks remained visible. Immediately after the masking interval, a mouse cursor looking exactly like the target appeared. In order to prevent the initial cursor position from acting as a confounding spatial reference, the cursor always appeared in the center of a landmark (if both landmarks were present, it appeared randomly in one or the other). The participants' task was to use the mouse cursor to reproduce the target's location as exactly as possible and to press the left mouse button when finished. The buttonpress elicited a 1000-Hz, 100-msec tone for feedback that the response had been registered. After an intertrial interval of $500 \mathrm{msec}$, a new trial began. Participants were in- 


\section{Possible landmark and target locations}

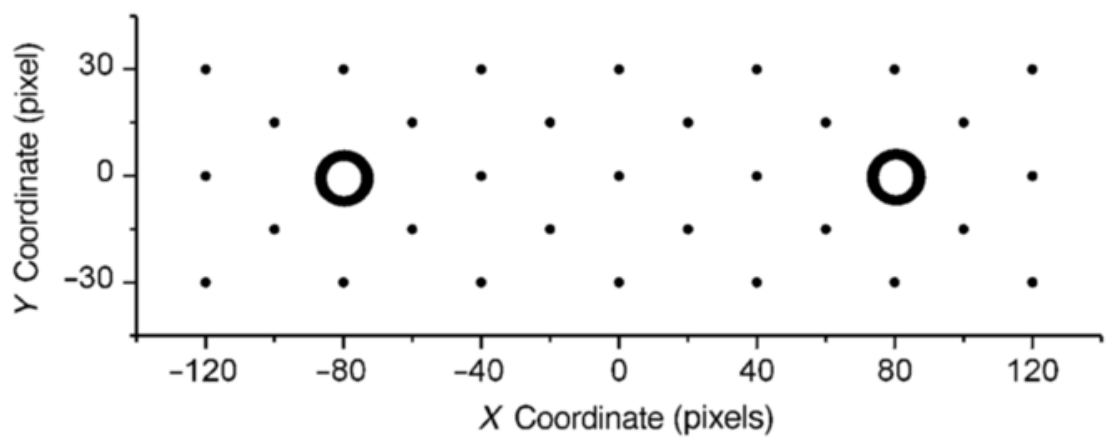

Time course of a trial

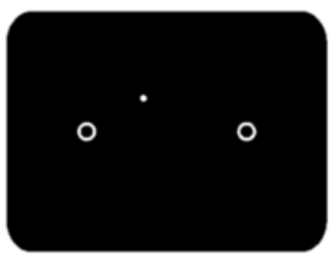

Target with one or two landmarks: $1,500 \mathrm{msec}$

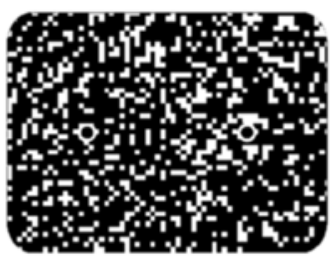

Dynamical mask (landmarks still visible): $500 \mathrm{msec}$

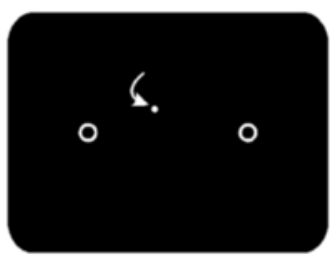

Reproduction of target location with mouse cursor

Figure 1. Upper panel: The spatial layout of landmarks and possible target locations. Note that either one or both landmarks could be presented, and targets never appeared at a position currently occupied by a landmark. Lower panel: time course of a trial. Stimuli appeared in color.

structed to work quickly, but it was emphasized that accuracy rather than speed was important in this task.

Stimulus conditions were counterbalanced in such a way that each combination of landmark configuration (left, right, both), starting position of the mouse cursor (left, right), and target position occurred quasi-randomly and equiprobably, with each combination appearing once every five blocks. After each block, participants received summary feedback about their average euclidean deviation from the true target position, rounded to the nearest pixel.

Participants performed three sessions of 15 blocks with 38 trials each. Each session started with an additional practice block of 38 trials, with stimuli drawn randomly from the experimental blocks. Practice trials were not analyzed. After the final session, participants were debriefed and received an explanation of the purpose of the experiment.

Data treatment and statistical methods. Trials with response times faster than $100 \mathrm{msec}$ or slower than 5,000 msec were excluded because these trials likely reflected anticipatory or guessing behavior. Trials with a target deviation larger than $30 \mathrm{px}$ in either the horizontal or vertical component were also excluded. From the remaining data, trials with horizontal or vertical error more than $3 S D s$ above or below the average deviation of a given participant were discarded. This procedure eliminated $5.76 \%$ of the raw data. Because participants differed considerably in the absolute magnitude of the distortional effects they produced, the $z$-standardized response distributions computed separately for each participant were entered into the analysis.

Both horizontal and vertical components of spatial distortions were included as dependent variables in a multivariate analysis of variance (MANOVA) with Wilks's ${ }_{\wedge}$ as a test statistic (Tabachnick \& Fidell, 1996). Readers unfamiliar with MANOVA can regard it as a straightforward extension of univariate analysis of variance, except that two-dimensional vectors rather than real numbers serve as the dependent variable. Repeated measures analyses were performed on the trimmed and standardized raw data by including a random participant factor (Maxwell \& Delaney, 2000). For simplicity, effects of the participant factor are not reported here.

\section{Results}

Figure 2 shows the distortional fields in the different landmark conditions. Distortions occurred in characteristic patterns depending on whether the left, the right, or both landmarks were present, displaying obvious regularity and symmetry.

In all conditions, the largest distortions occurred in the vicinity of the landmarks where reproductions were biased away from the landmark in a radial fashion. On the side of the display where no landmark was present, distortion was toward the landmark and away from the horizontal midline. Importantly, with both landmarks present, there was additional distortion away from the midpoint between the landmarks. MANOVA of the factors target position and landmark condition confirmed that the vectors of memory distortions strongly depended on target position $[\Lambda(60,298)=.393, p<.001]$. There was a main effect of landmark condition $[\wedge(4,18)=$ $.217, p=.006]$, mainly in the horizontal coordinate, which was due to the fact that the most prominent direction of 


\section{Experiment 1: Horizontal Landmarks}

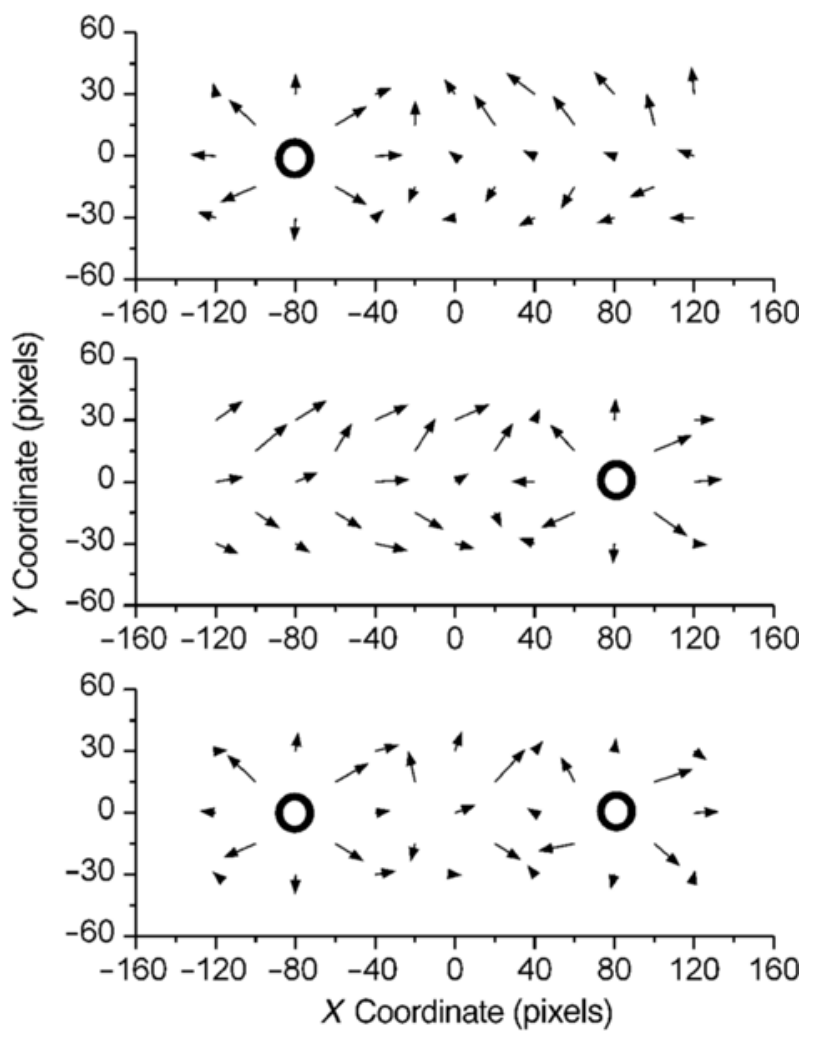

Figure 2. Distortional fields in the left-, right-, and bothlandmarks conditions in Experiment 1. Arrows point from the presented to the reproduced target position. Data were standardized before averaging across participants. Vector lengths were multiplied by a factor of 3 for better readability.

distortion was leftward in the left-landmark condition, rightward in the right-landmark condition, and neutral in the both-landmarks condition. Importantly, there was an interaction of target position and landmark condition $[\wedge(120,598)=.395, p<.001]$, confirming that the patterns of distortion differed across landmark conditions.

These effects can also be seen in Figure 3, where the horizontal and vertical components of distortion are plotted separately, each one averaged across the remaining coordinate (constant error, upper panel). Importantly, there is also a systematic pattern in the variance of reproductions (variable error, lower panel): Variable error is close to zero in the immediate vicinity of the landmarks but increases markedly with distance from the landmark. Thus, landmarks can be regarded as regions of minimum positional uncertainty.

The most striking feature of Figures 2 and 3 is that the local pattern of constant and variable error around a landmark is the same irrespective of whether the other landmark is present. Obviously, the left half of the bothlandmarks field is virtually identical to the left half of the left-landmark field (the right halves behaving analogously). This result strongly suggests a model in which each half-field is exclusively dominated by the nearest landmark and the influence of the farther landmark is zero-in other words, a model where landmarks partition the spatial representation into regions of interest. We formally tested this idea by defining a partition model that predicts the both-landmarks field from the singlelandmark fields according to the following equations:

$$
\begin{aligned}
& C_{L R}(x, y)=w_{L}(x, y) \cdot C_{L}(x, y)+w_{R}(x, y) \cdot C_{R}(x, y) ; \\
& V_{L R}(x, y)=w_{L}(x, y) \cdot V_{L}(x, y)+w_{R}(x, y) \cdot V_{R}(x, y),
\end{aligned}
$$

where $C_{L}(x, y), C_{R}(x, y)$, and $C_{L R}(x, y)$ are the vector patterns of constant error measured in the left-landmark, right-landmark, and both-landmarks conditions; $V_{L}(x, y)$, $V_{R}(x, y)$, and $V_{L R}(x, y)$ are the corresponding patterns of variable error; and $w_{L}(x, y)$ and $w_{R}(x, y)$ are weighting functions, defined as

$$
\begin{aligned}
& x<0: w_{L}(x, y)=1 ; w_{R}(x, y)=0 ; \\
& x=0: w_{L}(x, y)=w_{R}(x, y)=0.5 ; \\
& x>0: w_{L}(x, y)=0 ; w_{R}(x, y)=1 .
\end{aligned}
$$

This model does nothing more than putting together the left half of the left-landmark field and the right half of the right-landmark field in a cut-and-glue operation. Constant and variable error on the vertical midline $(x=0)$ are handled by averaging the midline effects in the singlelandmark fields.

Comparisons of observed and predicted values of distortion are shown in Figure 4. The partition model provides an excellent fit to both constant and variable error, except for the midpoint, where it slightly underestimates the bias and overestimates the variance associated with it. Linear regression of observed against predicted means confirmed good fits for both constant error [horizontal, $F(1,11)=66.62, p<.001, R^{2}=.858$; vertical, $F(1,3)=$ $38.82, p=.008, R^{2}=.928$ ] and variable error [horizontal, $F(1,11)=28.58, p<.001, R^{2}=.722$; vertical, $F(1,3)=$ $\left.196.12, p=.001, R^{2}=.985\right]$, each regression being statistically indistinguishable from a perfect fit with slope of 1 and intercept of 0 .

\section{Discussion}

Experiment 1 replicates earlier findings that spatial visual memory for briefly presented targets is distorted by landmark stimuli, following a complex but regular and symmetrical pattern. The major features of this pattern are locally restricted radial distortions away from the landmarks, distortions away from the horizontal midline and toward the landmarks in the rest of the display, and additional distortion from the midpoint between the landmarks. Besides being sources of spatial biases, landmarks also provide regions of low spatial uncertainty, as shown by markedly reduced variable error. The pattern of spatial biases around the horizontal midline might indicate a special role for the allocentric horizontal axis or an influence of the imaginary line connecting the landmarks. Alternatively, it might be due in part to the visible monitor frame or some further spatial cues available during the experiment. 


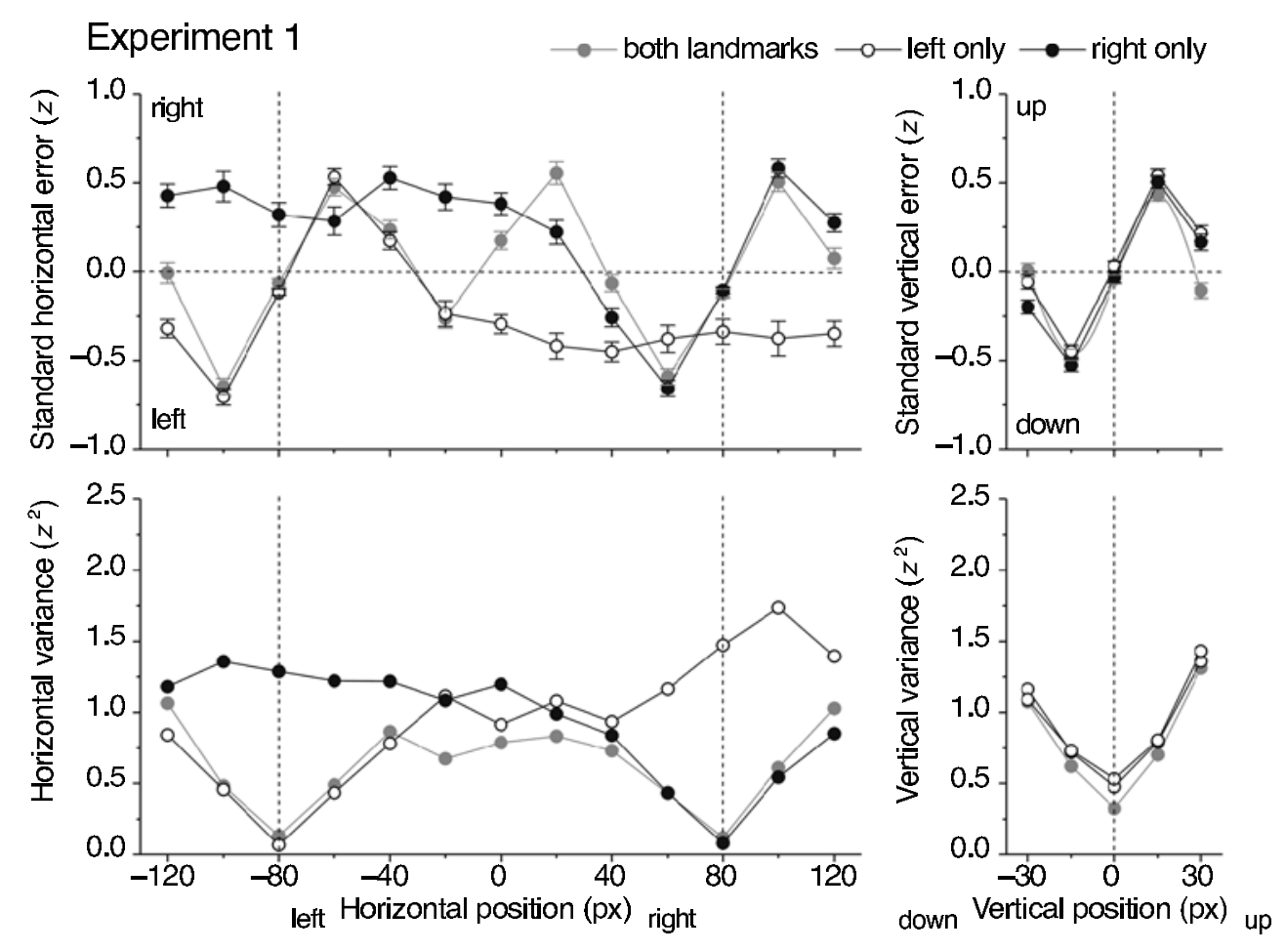

Figure 3. Average spatial distortions in Experiment 1 for the left-, right-, and both-landmarks conditions. Upper left: systematic error in horizontal direction averaged over vertical positions. Upper right: systematic error in vertical direction averaged over horizontal positions. Lower left: horizontal variable error. Lower right: vertical variable error. Data were standardized before averaging across participants.

Distortions in the both-landmarks condition are well fit by a simple partition model that assumes that both constant and variable error can be predicted by considering only local effects around each single landmark. Following this model, distortions in the left and right halves of the both-landmarks field are identical to the left half of the left-landmark field and to the right half of the right-landmark field, respectively, clearly indicating that local effects around each landmark are invariant when a second landmark is added at some distance.

However, the partition model makes wrong predictions for the targets located directly at the vertical midline, the partition boundary. Here, spatial bias is slightly underestimated whereas variable error is overestimated. This pattern is consistent with the midpoint between the landmarks being another region of low spatial uncertainty or "virtual landmark" that, like the actual landmarks, might have a repulsory effect of its own.

\section{EXPERIMENT 2}

In Experiment 1, landmarks and targets were presented on a customary computer monitor, with the monitor edges clearly visible. Thus, participants might have used spatial reference stimuli other than the landmarks to encode the location of the target dot. The laboratory setup was therefore modified to reduce these possible ar- tifacts as effectively as possible. Participants were seated at the wider end of a large funnel, with the monitor situated at the narrow end so that the laboratory environment was blocked from view and the monitor edge was circular rather than rectangular. Because the experiment was performed in darkness, and brightness masking was used to keep the participants light adapted throughout the session, the circular monitor edges were clearly visible only during the masking interval and almost invisible during the presentation of landmarks and target.

Apart from improving the control of visual presentation, vertical in addition to horizontal configurations of landmarks were used to check for orientational invariance of distortional fields. Most important, this was also a critical test of whether the partition model from Experiment 1 could be generalized to a new orientation of the landmark configuration.

\section{Method}

Participants. Eight more students (age 21 to 26, 7 of them female, all of them right-handed) participated for course credit or for a payment of $15 \mathrm{DM}$ per hour. Their vision was normal or corrected to normal.

Apparatus. The electronic equipment was the same as in Experiment 1 . The funnel was made of a homogeneously textured fabric and left only a circular central portion $\left(17 \mathrm{~cm}, 8.84^{\circ}\right)$ of the screen where stimuli were presented at a viewing distance of $110 \mathrm{~cm}$ so that $1^{\circ}$ of visual angle equaled approximately $32 \mathrm{px}$. The 


\section{Experiment $1 \multimap$ observed}
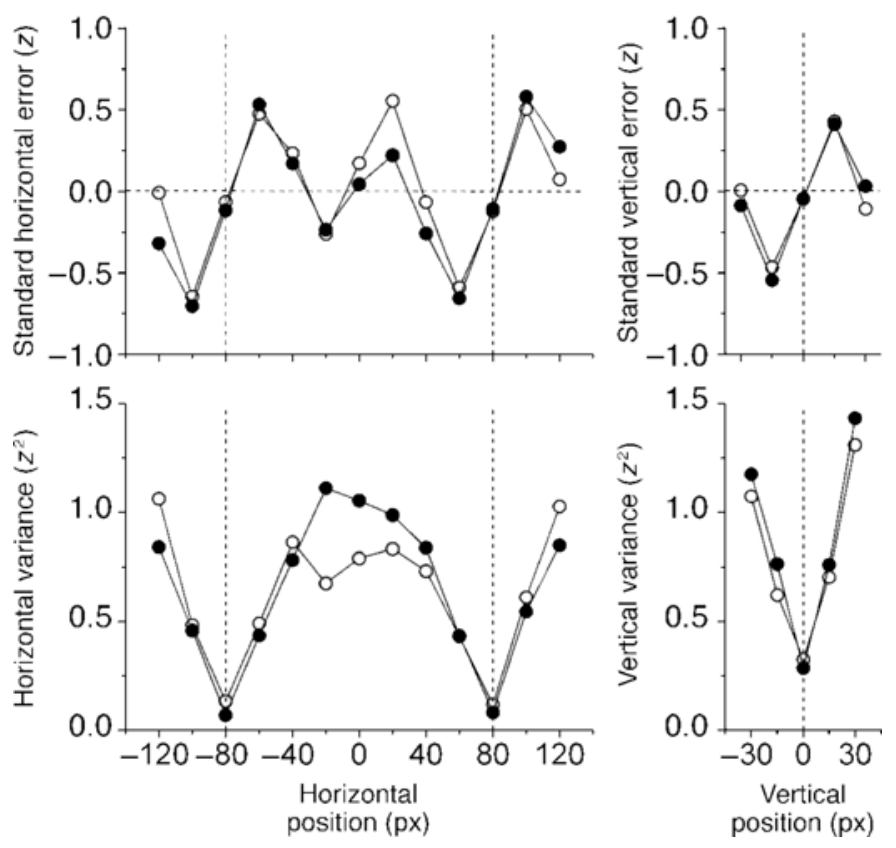

Figure 4. Predictions of the partition model for systematic (upper panel) and variable error (lower panel) in Experiment 1.

laboratory environment was completely blocked from view. The experiment was performed in darkness, with the lighting switch within reaching distance of the participant.

Stimuli. As before, all stimuli were presented against a black background $\left(0.01 \mathrm{~cd} / \mathrm{m}^{2}\right)$. Landmarks were green unfilled circles $\left(2.95 \mathrm{~cd} / \mathrm{m}^{2}\right), 9 \mathrm{px}\left(.26^{\circ}\right)$ in diameter and $64 \mathrm{px}\left(1.82^{\circ}\right)$ to the left or right of the screen center. The target was a small gray dot $\left(10.8 \mathrm{~cd} / \mathrm{m}^{2}\right)$ with a diameter of $3 \mathrm{px}\left(.09^{\circ}\right)$. The grid of possible targets was similar to that used in Experiment 1, but the overall size of the configuration was scaled to approximately $73 \%$ to accommodate the restricted display size, now spanning a visual angle of about $6.36^{\circ} \times$ $1.36^{\circ}$. In addition to the horizontal configuration of landmarks and targets, there was a vertical configuration where the landmarks and the grid of possible target locations were rotated by $90^{\circ}$. Instead of the dynamical noise mask, a simple brightness mask was used where the whole display was set to white $\left(43.0 \mathrm{~cd} / \mathrm{m}^{2}\right)$. A previous experiment had shown that the brightness mask was as effective as the dynamic mask, and it had the additional advantage of keeping the participants light-adapted during the experiment so that the circular monitor edges were hardly visible. It also excluded the potential strategy of using the black and white mask elements as local spatial cues. As before, the landmarks remained visible during the masking interval.

Procedure. The time course of events within a trial was identical to that of Experiment 1. The only exception was that the mouse cursor no longer started from one of the two landmark positions but randomly from one of eight positions arranged regularly around the center of the display at a radius of $120 \mathrm{px}\left(3.10^{\circ}\right)$. The cursor was not visible unless moved by at least $15 \mathrm{px}\left(.39^{\circ}\right)$. This manipulation assured that the initial cursor position was unpredictable, which had not been the case in the single-landmark conditions of Experiment 1 .

Apart from these changes in stimulus conditions, the procedure was the same as in Experiment 1. Participants performed four sessions of 15 blocks with 38 trials each. Each session started with an additional practice block of 38 trials. After the final session, par- ticipants were debriefed and received an explanation of the purpose of the experiment.

\section{Results}

Data treatment proceeded as described in Experiment 1 . The trimming procedure eliminated $3.05 \%$ of the raw data.

As observed in Experiment 1, distortional fields in the vicinity of single landmarks were very similar to the corresponding regions in the both-landmarks conditions (Figure 5): Biases and variances around a given landmark were the same whether or not the other landmark was present. In both horizontal and vertical conditions, reproduced target locations in the vicinity of landmarks were biased away from the landmarks. As observed in Experiment 1, there was additional distortion away from the midpoint of the two-landmark configuration. However, elimination of monitor edges as a spatial reference frame seems to have led to additional biases that span the entire distortional field: There is a conspicuous downward bias (in allocentric coordinates) that is especially pronounced in the vertical condition when only the lower landmark was presented.

For the horizontal condition, there was a main effect of target position on memory distortion $[\wedge(60,418)=$ $.329, p<.001]$, but not of landmark condition $[\wedge(4,26)=$ $.662, p=.235]$. Importantly, patterns differed across landmark conditions, as shown by a significant interaction of target position and landmark condition $[\wedge(120,838)=$ $.431, p<.001]$. In the vertical condition, there was also 


\section{Exp. 2 - horizontal landmarks}
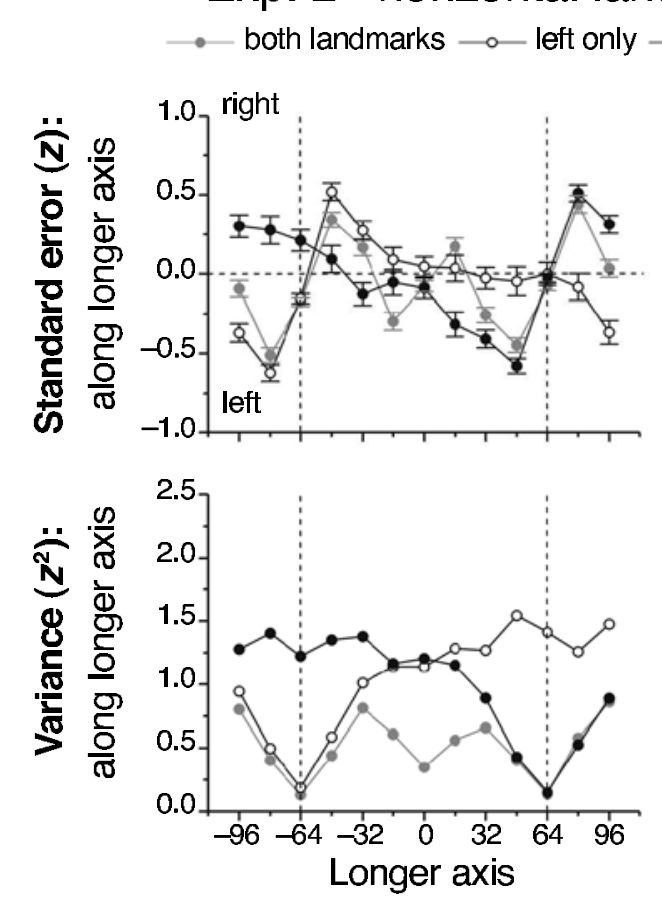

Exp. 2 - vertical landmarks

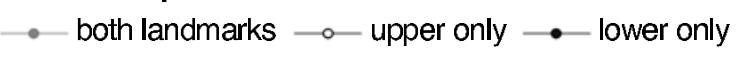

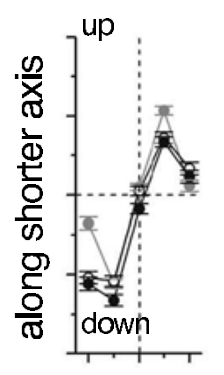
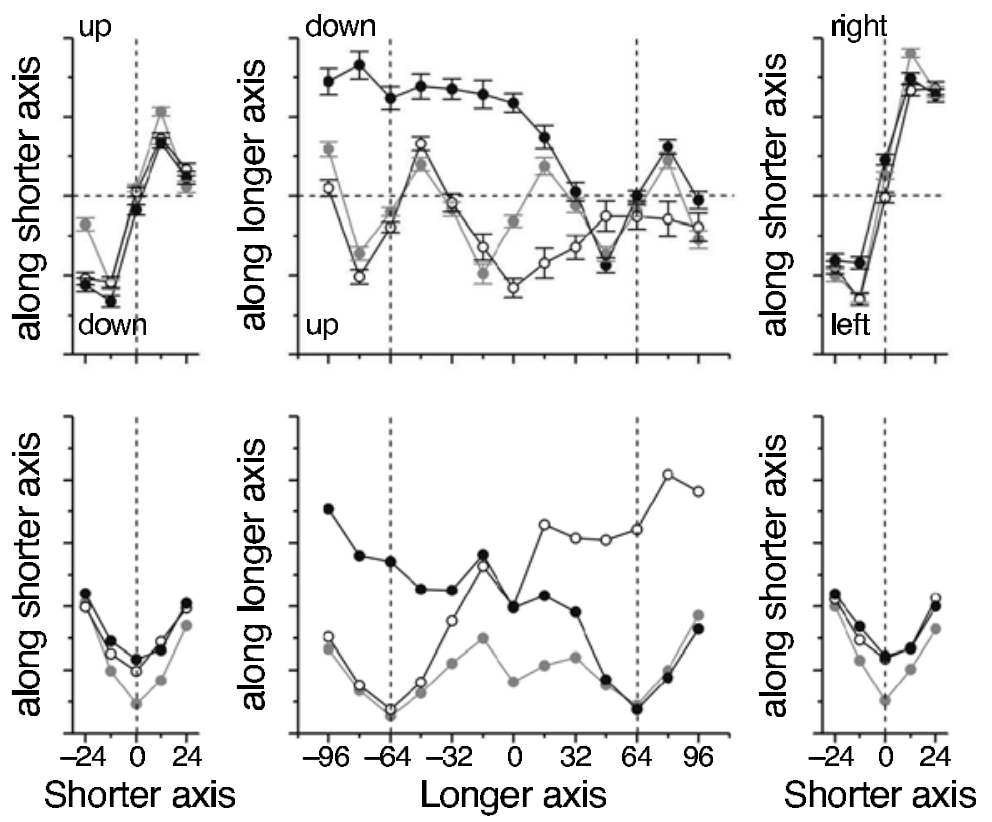

Figure 5. Systematic and variable error for horizontal and vertical landmark configurations in Experiment 2. Data were standardized before averaging across participants.

a main effect of target position $[\wedge(60,418)=.190, p<$ $.001]$, as well as an interaction of target position and landmark condition $[\wedge(120,838)=.565, p<.001]$. However, there was also a main effect of landmark condition $[\wedge(4,26)=.352, p=.007]$, significant only in the vertical component of distortion and mainly due to the fact that the most prominent direction of distortion was upward in the upper-landmark condition, downward in the lowerlandmark condition, and neutral in the both-landmarks condition.

Figure 6 shows a comparison of the effects obtained in the both-landmarks conditions, with the predictions of the partition model applied separately for horizontal and vertical configurations. For the horizontal configuration, linear regression showed that the fit was good for the shorter axis of the display but not for the longer axis, for constant error [longer axis, $F(1,11)=20.18, p=.001$, $R^{2}=.647$; shorter axis, $F(1,3)=74.79, p=.003, R^{2}=$ .949] as well as variable error [longer axis, $F(1,11)=9.27$, $p=.011, R^{2}=.457$; shorter axis, $F(1,3)=13.22, p=$ $\left..036, R^{2}=.815\right]$, the regression slopes for long-axis effects now being significantly lower than 1 . This reduced fit was due to the fact that the partition model now failed to predict the bias away from the midpoint and greatly overestimated variable error at the midpoint. In the vertical configuration, the partition model provided a good fit for constant error [longer axis, $F(1,11)=28.81, p<$ $.001, R^{2}=.724$; shorter axis, $F(1,3)=219.73, p=.001$,
$R^{2}=.987$ ] but was slightly worse for variable error [longer axis, $F(1,11)=12.80, p=.004, R^{2}=.538$; shorter axis, $\left.F(1,3)=15.53, p=.029, R^{2}=.838\right]$, where the regression slope for longer-axis variable error was significantly lower than 1 . Here again, this was mainly due to a marked overestimation of variable error at the midpoint.

The generally good fit of the partition model suggests that visual landmarks mutually restrict their spatial influence rather than exerting their influence independently. Importantly, this type of interaction rules out all types of linear models, which would assume that distortional fields of single landmarks simply add, perhaps after being weighted by some constant. If distortional fields combined linearly, this would be theoretically important because it would allow for the prediction of arbitrarily complex configurations of landmarks from their single-landmark elements. For our purposes, the most flexible model of this sort would be a two-parameter linear model, which employs Equation 1 with modified weighting functions:

$$
\begin{aligned}
& w_{L}(x, y)=a ; \\
& w_{R}(x, y)=b ;
\end{aligned}
$$

with $a$ and $b$ constant. A special case is the superposition model, with $a=b=1$, which assumes that the distortional effects of single landmarks simply superimpose, just like physical force fields do (e.g., electromagnetic or gravitational fields). 
Exp. 2 - horizontal landmarks
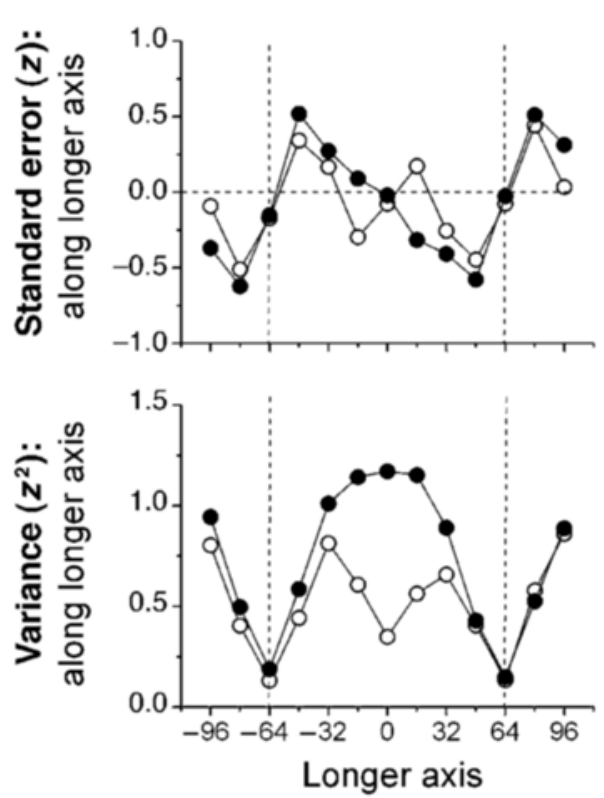

Exp. 2 - vertical landmarks

$\multimap$ observed $\longrightarrow$ partition model
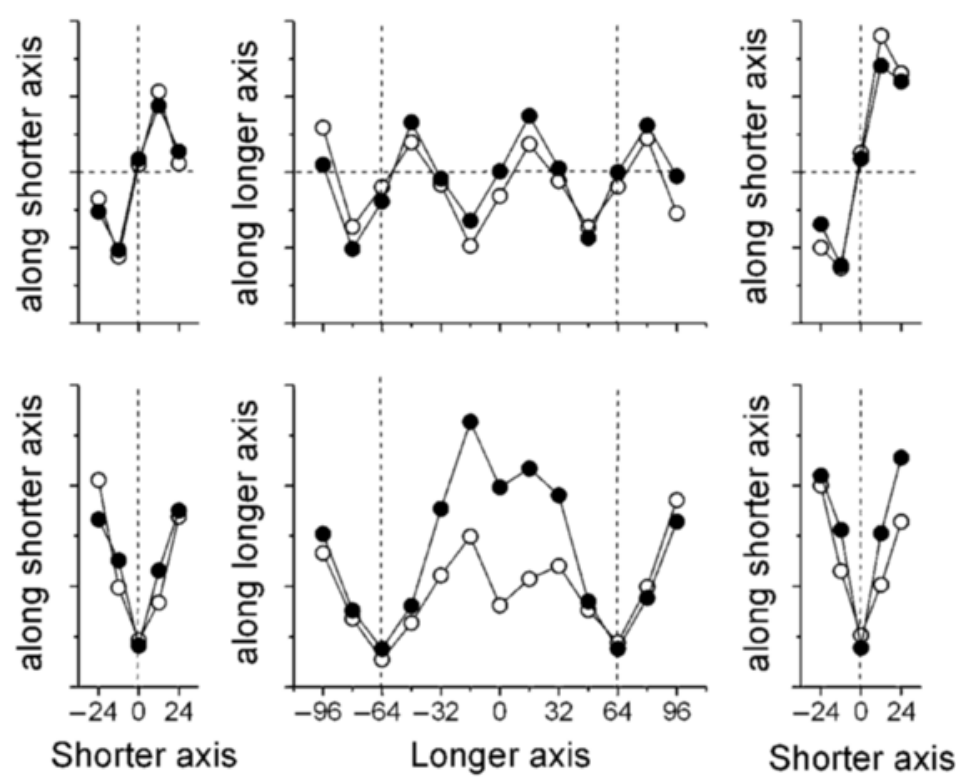

Target position (px)

Figure 6. Predictions of the partition model for systematic (upper panels) and variable error (lower panels) in Experiment 2, plotted separately for horizontal (left panels) and vertical (right panels) landmark configurations.

Figure 7 shows the results of fitting the constant-error predictions of the two-parameter linear and superposition models to the observed data (predictions of variable error are possible only under additional assumptions and are omitted here). It is obvious that the superposition model tends to grossly overestimate the amount of distortion because it adds distortions from those display halves containing landmarks to those where no landmarks were presented-in a way, it spoils the fit of the partition model by adding unsystematic distortions from the no-landmark halves. In contrast, the two-parameter linear model (fitted by least-squares methods) underestimates distortions because it best fits the data with rather small weighting parameters (average $a=0.388$; average $b=0.317$ ), reflecting a compromise between an optimal weight near 1 in the hemifield containing the landmark and a weight near 0 on the opposite side. $R^{2}$ values for both models are unsatisfactory (average $R^{2}=$ .386), especially for fit along the longer axes. Most importantly, both linear models make qualitatively wrong predictions: They predict distortions toward the midpoint rather than away from it, and the superposition model predicts marked outward distortion directly at the landmark locations, where no such distortion is observed.

\section{Discussion}

Distortional fields of horizontal and vertical configurations of two landmarks are similar to those observed in
Experiment 1, with distortion away from the landmarks and midpoint and reduced variable error at the landmarks. The reduction in variable error at the midpoint is more conspicuous than in Experiment 1 and strongly suggests that the midpoint is another region of low spatial uncertainty, or "virtual landmark." In addition, there is some global downward bias that is especially prominent when only a lower landmark is presented. This might be a result of the changed viewing conditions: In Experiment 1 , the presence of an extrinsic rectangular reference system defined by the monitor frame might have helped neutralize the downward bias and at the same time might have induced encoding of single-landmark fields in monitor-aligned coordinates, thereby enhancing symmetry of distortional fields. Such an interpretation is also suggested by the fact that the downward bias is eliminated when a second, upper landmark is provided as an additional spatial reference.

Despite its simplicity, the partition model fits these data very well in most regions of the display, clearly indicating that local effects around each landmark are invariant when a second landmark is added at some distance. However, this model is not able to account for effects directly on the partition boundary, markedly overestimating variable error and failing to predict repulsion from the midpoint in the horizontal condition. Again, this suggests that the midpoint has repulsory effects on memory representations similar to those of the visible landmarks. 


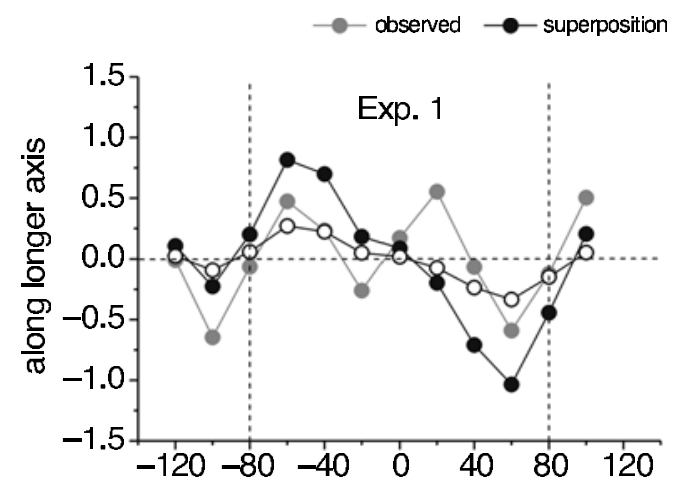

- 2-parameter linear
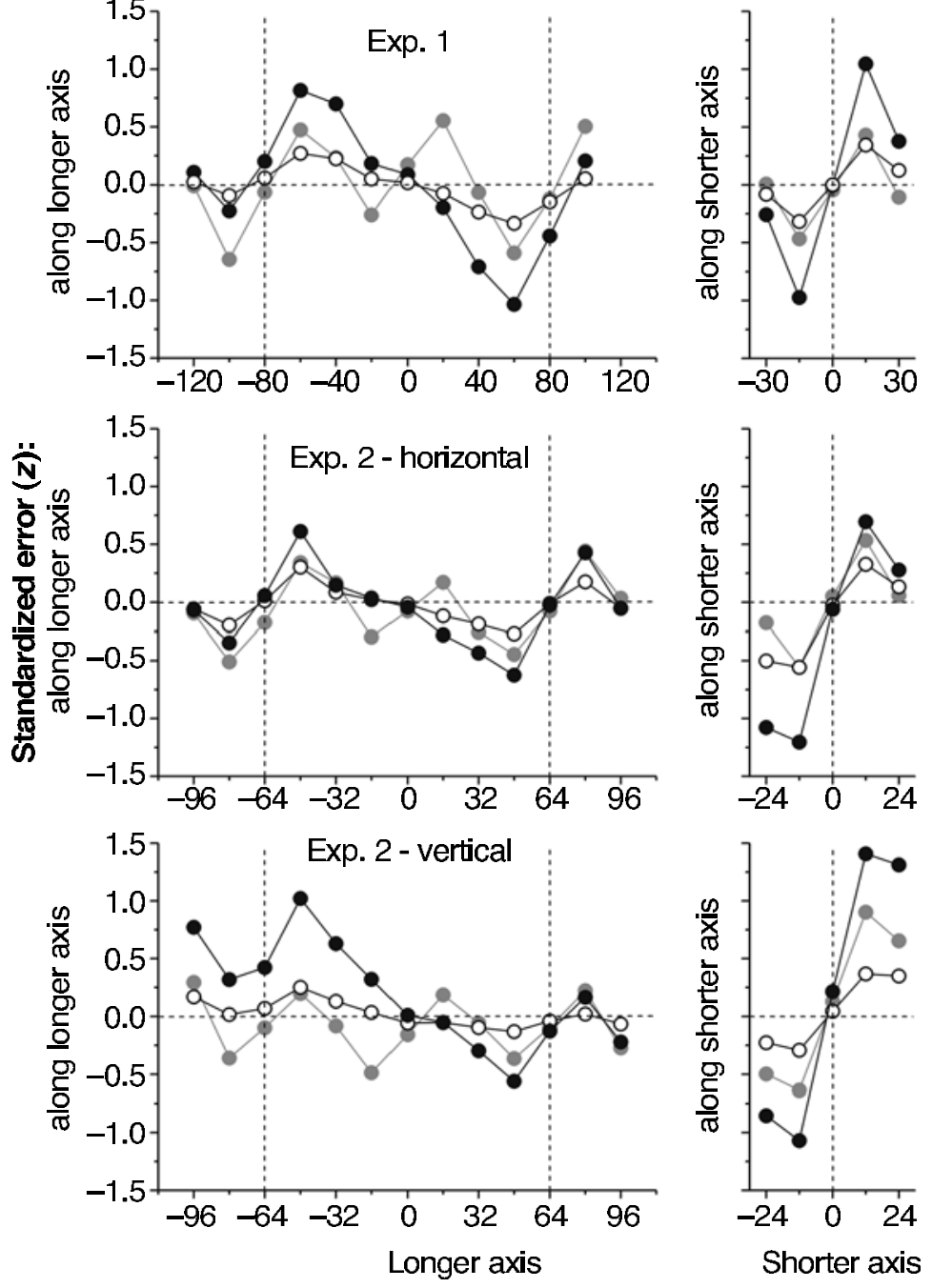

Target position (px)

Figure 7. Predictions of the linear (two-parameter and superposition) models for systematic error in the two-landmark conditions of Experiments 1 and 2.

\section{GENERAL DISCUSSION}

Landmarks in the visual field generate a systematic pattern of spatial memory distortions, with target positions remembered too far away from the landmarks and (in two-landmark displays) from the midpoint between the landmarks. Targets further away from the landmarks follow different patterns. In Experiment 1, where the monitor frame could be used as an additional spatial reference, they were distorted away from the longer midline and toward the landmark in a fishbone-like pattern. In Experiment 2, where such spatial cues were eliminated, there was an additional global downward bias. The visibility of the rectangular monitor frame seems to have stabilized the distortional patterns by removing un- certainty about the position and orientation of allocentric axes, especially when only one landmark was present. In contrast, presentation of $t w o$ landmarks seems sufficient for establishing a stimulus-based reference system that is not affected by the presence or absence of the monitor frame.

Distortional fields of two landmarks can be predicted largely from those of the single landmarks by adopting the principle that distortions are governed only by the nearest landmark and are locally invariant when a second landmark is introduced. Thus, our data strongly suggest a partition principle of two-landmark displays where the memory representation is subdivided into regions invariably dominated by single landmarks. This principle rules out any kind of linear model assuming 
that single-landmark fields combine by superposition or weighted summation.

However, even the partition model cannot explain all aspects of the data, because it does not reliably predict distortion from the midpoint. Unpredicted by the model, the midpoint is a point of minimum variable error in all three configurations reported here, which suggests that the midpoint serves as an additional spatial referencea virtual landmark creating its own pattern of distortion. This is in line with an elegant psychophysical model by Hollands and Dyre (2000) showing that when observers strategically code stimuli relative to some anchor points on a psychophysical continuum, this creates a cyclical pattern of biases away from these anchor points similar to the one observed here. Bryant and Subbiah (1993) have also shown that the pattern of spatial memory distortions strongly depends on which kind of coordinate frame is strategically used by the observers during encoding.

Importantly, our concept of virtual landmarks is at odds with models assuming that visual stimuli generate distortions in a purely bottom-up fashion, without taking into account the emerging geometrical properties of the ensuing figures. One example for an entirely stimulusdriven model of spatial distortions is the one proposed by Watson (1977) for visual illusions, which assumes that visual stimuli distort perceived space in strict analogy to how mass points distort physical space in Einsteinian physics. Such a physical analogy is also at odds with the partition principle stating that distortional fields in spatial memory do not combine linearly, as physical force fields do.

Although Nelson and Chaiklin's (1980) model correctly states that distortional fields combine after being weighted with a function that declines with increasing distance from the landmark, it is not recognized that this weighting function approximates a simple step function: The model's assumption that landmarks exert their influence independently across the entire display is at odds with the partition principle. What's worse, Nelson and Chaiklin's model incorrectly assumes that spatial memory is biased toward a landmark, not away from it, and that distortion becomes stronger with increasing distance from the landmark. From our point of view, a simpler and more intuitive explanation of their data would be that remembered target positions were strongly distorted away from the midpoint of their circular display, which acted as a virtual landmark.

The Huttenlocher et al. (1991) model is able to account for surprising "Gestalt-like" effects when a landmark is added to a preexisting configuration because it assumes that participants parse the display into new spatial categories. Although it flexibly accounts for different patterns of constant error, however, it implies predictions about variable error that are at odds with our data. The model would account for the two-landmark data pattern by suggesting that the spatial representation is segmented into categories along the horizontal midline: between left landmark and midpoint, between right landmark and midpoint, and beyond the landmarks on either side. The horizontal midline itself would be assumed to divide each of these categories into an upper and a lower part. Distortions from the landmarks and midpoint would then be explained by assuming (1) that category membership at these points is uncertain because they fall on category boundaries, (2) that the locations of these boundaries themselves are uncertain, and (3) that prototype locations are used to assist in reconstruction of uncertainly remembered target locations, which leads to bias toward the category centers. Although this model could deal well with the pattern of constant error, its predictions for variable error would be the opposite of the observed pattern. Because category membership of a target on a boundary is uncertain, these locations should be associated with maximum variance in reproductions; at the same time, prototype locations should be associated with minimum variance. Both predictions are inconsistent with our results.

We therefore argue that the concept of prototype locations should be abandoned in favor of a model assuming repulsory effects on spatial memory from regions of low spatial uncertainty, which might be given explicitly by visual landmarks or implicitly by auxiliary constructions of connecting lines and midpoints. Independent evidence for the importance of such virtual points and lines comes from experiments by Psotka (1978). Each participant had the simple task of arbitrarily placing a single dot anywhere in an outline figure presented on a sheet of paper. Strikingly, with large groups of participants, very systematic patterns emerged depending on the geometry of the outline figure: People tended to place their dots on imaginary lines connecting salient points in the display as well as on their perpendiculars. We informally employed this approach with students from different sections of an introductory psychology course at the University of Idaho. Each student received a set of six letter-sized cards, one of which showed two single dots of $3.5-\mathrm{mm}$ diameter printed $100 \mathrm{~mm}$ apart from each other in either a horizontal configuration or tilted by plus or minus $15^{\circ}$. The remaining cards showed different stimuli that are not of interest here. Participants were tested collectively, each one receiving the cards in the same order. The instruction was to "draw a small dot at any location you feel 'goes well' with the figure. Wherever you want to put it is fine-there is no correct answer." Of 304 students, 303 produced valid results, which have been superimposed after appropriate rotation in Figure 8. Strikingly, most participants placed their dots on the midpoint, the vertical midline, or the virtual line connecting the landmarks - that is, those points and lines that are found to have repulsory effects on spatial memory. This also implies that preferences in the placement of a dot within the stimulus display cannot account for the distortions found in our studies.

We argue that visible landmarks as well as the virtual points and lines constructed from them are the actual generators of spatial distortions because they tend to 


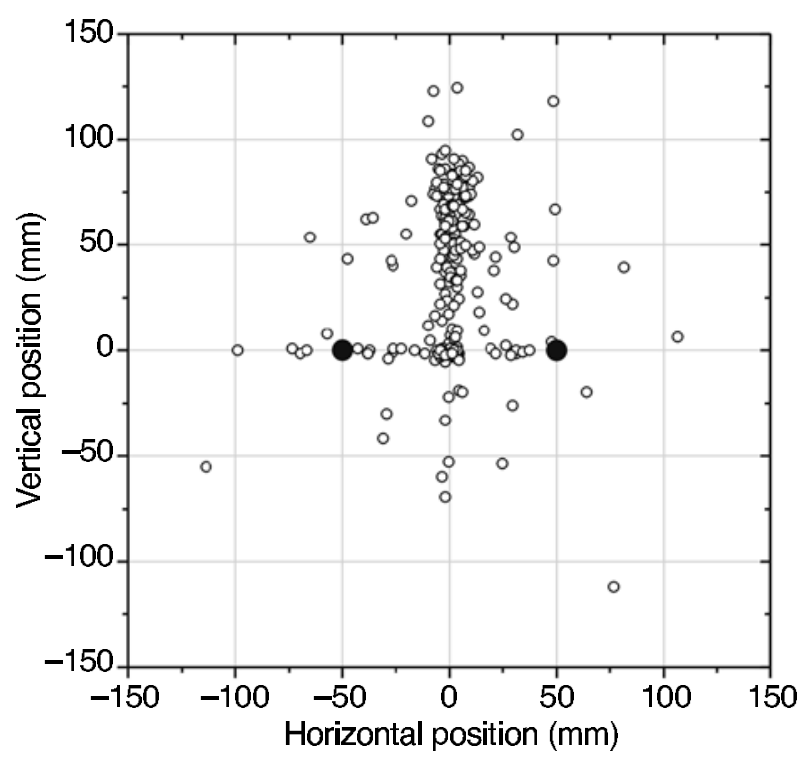

Figure 8. Results from asking $\mathbf{3 0 3}$ participants to arbitrarily put a pencil dot anywhere on a single sheet of paper showing a two-landmark configuration. Results from different orientations of the figure (varied between subjects) are aligned and superimposed. Landmark dots are shown in black. Grid lines were not present in the original stimulus.

bind overt or covert visual attention. Our results are generally consistent with the model proposed by Suzuki and Cavanagh (1997), which predicts strictly regional distortions away from the focus of visual attention by pointing out how lateral interactions between attended and unattended receptive fields (Desimone \& Duncan, 1995; Gilbert, 1998) would bias the spatial representation. Their proposed mechanisms of one-dimensional perceptual distortions might be adapted for two-dimensional spatial memory tasks-for example, by considering topographical memory representations like those identified in dorsolateral prefrontal cortex (e.g., Camperi \& Wang, 1998; Funahashi, Bruce, \& Goldman-Rakic, 1989). In particular, attention might be operating directly on spatial memory maps, or these memory maps could inherit spatial biases from more perceptual representations as participants continue to attend to the landmarks during the retention interval. In the long run, visual landmarks will tend to attract overt or covert visual attention more than other regions of the display; in addition, attention could be strategically deployed to salient regions like connecting lines or midpoints acting as virtual landmarks. It can be shown that manipulations of spatial attention strongly influence the pattern of spatial memory distortions (Trommershäuser \& Schmidt, 2003).

Because each stimulus in the visual field might be a generator of spatial distortions, the partition principle has an important function for preventing multilandmark systems from catastrophic cumulation of variable error. In our view, the deployment of visual attention creates local minima of spatial uncertainty. Although this pro- cess introduces spatial biases, the advantages coming from reduced spatial uncertainty outweigh the relatively small distortions introduced in the process (Huttenlocher et al., 1991), because uncertainty minima are immune to distortions generated elsewhere in the display. Visual attention creates a dense net of virtual points and lines that can be predicted on the basis of stimulus geometry and helps to conserve the fidelity of the spatial representation. In a visual field normally cluttered with spatial cues, these principles provide stability to plastic, attentionally modulated topographic memory maps that might otherwise perish in accumulating noise. Similar principles might hold for topographical representations in other sensory domains.

\section{REFERENCES}

Bryant, D. J., \& Subbiah, I. (1993). Strategic and perceptual factors producing tilt contrast in dot localization. Memory \& Cognition, 21, 773-784.

CAMPERI, M., \& WANG, X. J. (1998). A model of visuospatial working memory in prefrontal cortex: Recurrent networks and cellular bistability. Journal of ComputationalNeuroscience, 5, 383-405.

Crawford, L. E., Huttenlocher, J., \& Engebretson, P. H. (2000). Category effects on estimates of stimuli: Perception or reconstruction? Psychological Science, 11, 280-284.

Desimone, R., \& Duncan, J. (1995). Neural mechanisms of selective visual attention. Annual Review of Neuroscience, 18, 193-222.

Diedrichsen, J. (1998). Gibt es ein motorisches Kurzzeitgedächtnis für Positionen im Greifraum? Unpublished thesis, University of Göttingen.

Diedrichsen, J., Werner, S., Schmidt, T., \& TrommershäUSER, J. (in press). Immediate spatial distortion of pointing movements induced by visual landmarks. Perception \& Psychophysics.

Flanders, M., Helms Tillery, S. I., \& Soechting, J. F. (1992). Early stages in a sensorimotor transformation. Behavioral \& Brain Sciences, 15, 309-362.

Funahashi, S., Bruce, C. J., \& Goldman-Rakic, P. S. (1989). Mnemonic coding of visual space in the monkey's dorsolateral prefrontal cortex. Journal of Neurophysiology, 61, 331-349.

Gilbert, C. D. (1998). Adult cortical dynamics. Physiological Review, 78, 467-485.

Hollands, J. G., \& Dyre, B. P. (2000). Bias in proportion judgments: The cyclical power model. Psychological Review, 107, 500-524.

Huttenlocher, J., Hedges, L. V., \& Duncan, S. (1991). Categories and particulars: Prototype effects in estimating spatial location. Psychological Review, 98, 352-376.

Laeng, B., Peters, M., \& McCabe, B. (1998). Memory for locations within regions: Spatial biases and visual hemifield differences. Memory \& Cognition, 26, 97-107.

Maxwell, S. E., \& Delaney, H. D. (2000). Designing experiments and analyzing data: A model comparison perspective. Mahwah, $\mathrm{NJ}$ : Erlbaum.

Nelson, T. O., \& Chaiklin, S. (1980). Immediate memory for spatial location. Journal of Experimental Psychology: Human Learning \& Memory, 6, 529-545.

Palmer, S. E. (1999). Vision science: Photons to phenomenology. Cambridge, MA: MIT Press.

PsotKA, J. (1978). Perceptual processes that may create stick figures and balance. Journal of Experimental Psychology: Human Perception \& Performance, 4, 101-111.

Schmidt, T. (2002). Spatial distortions in visual short-term memory. Electronically published doctoral dissertation, University of Göttingen. Available: http://webdoc.sub.gwdg.de/diss/2002/schmidt_thomas/ schmidt_thomas.pdf.

Sheth, B. R., \& Shimojo, S. (2001). Compression of space in visual memory. Vision Research, 41, 329-341.

Suzuki, S., \& CaVAnagh, P. (1997). Focused attention distorts visual 
space: An attentional repulsion effect. Journal of Experimental Psychology: Human Perception \& Performance, 23, 443-463.

Taвachnick, B. G., \& Fidell, L. S. (1996). Using multivariate statistics (3rd ed.). New York: HarperCollins.

TrommershäUSER, J., \& SCHmidt, T. (2003). Attention controls spatial distortions in visual short-term memory. Manuscript in preparation.

Watson, A. I. (1977). A theory of visual illusions. British Journal of Mathematical \& Statistical Psychology, 30, 43-59.

Werner, S., \& Diedrichsen, J. (2002). The time course of spatial memory distortions. Memory \& Cognition, 30, 718-730.
Werner, S., \& Schmidt, T. (2000). Investigating spatial reference systems through distortions in visual memory. In C. Freksa, W. Brauer, C. Habel, \& K. F. Wender (Eds.), Spatial cognition II: Integrating abstract theories, empirical studies, formal methods, and practical applications (pp. 169-183). Berlin: Springer-Verlag.

(Manuscript submitted for publication October 17, 2001; revision accepted for publication February 13, 2003.) 